\title{
Diagnóstico de las competencias lectoescritas en estudiantes de posgrado ${ }^{1}$
}

\author{
LUZ MARINA TRIANA MURILLO²
}

\section{ESCUELA DE POSGRADOS DE LA FUERZA AÉREA COLOMBIANA}

Recibido, octubre 09 de 2014

Concepto evaluación, noviembre 11 de 2014

Aceptado, enero 21 de 2015
Referencia: Triana Murillo, L.M. (2015). "Diagnóstico de las competencias lectoescritas en estudiantes de posgrado". Revista Academia y Virtualidad, 8, (1), 76-87

\section{Resumen}

Este artículo resulta de la investigación "Didáctica para el desarrollo de las competencias lectoescritas en estudiantes de posgrado", el cual aborda la problemática del nivel de apropiación de las competencias en lectoescritura por parte de estudiantes de educación terciaria, en posgrado. La investigación determina las causas del índice de no graduados en una institución de Educación Superior y el estado de las competencias en lectoescritura de los estudiantes de la especialización de Logística Aeronáutica a través de un estudio de caso, en el cual se evidencia que el $85 \%$ de la muestra se encuentra en niveles de regular a deficiente, clasificando a los estudiantes en el nivel Al de competencia en el dominio de su lengua materna de acuerdo con los estándares del Marco Común Europeo.

Palabras clave: competencias, lectoescritura, posgrados, Educación Superior.

\section{Reading and writing skills assessment of post-grade students}

\begin{abstract}
This paper is a result of a research, "Didactics to develop reading-writing skills of post-grade students", whose problem is the level of reading and writing proficiency by postgraduate students. It shows the causes of the undergraduates in an institution of higher education and the state of reading and writing skills by Aeronautics Logistics specialization students in a case study, which evidences that 85 percent of the sample has moderate-to-poor levels, ranking them in level A1 of their native language according to the Common European Framework standards.
\end{abstract}

Keywords: skills, reading and writing skills, postgraduate, higher education.

1. Artículo de investigación.

2. Docente, escritora e investigadora, especialista en tendencias actuales de Literatura, especialista en docencia universitaria, magíster en educación, docente y asesora de investigación, Escuela de Postgrados, Fuerza Aérea Colombiana. Contacto: luz.triana@ima.edu.co 
Luz Marina Triana Murillo

\section{Diagnóstico das competências leto-escritas em estudantes de pós-graduação}

\section{Resumo}

Este articulo é o resultado da pesquisa "Didática para o desenvolvimento das competências leto-escritas em estudantes de pós-graduação", o qual aborda a problemática do nível de apropriação das competências em leto-escritura pelos estudantes de educação terciária, no pós-grau. A pesquisa estabelece as causas raiz do índice de não graduados em uma instituição de Educação Superior e o estado das competências em leto-escritura dos estudantes da especialização de Logística Aeronáutica através de um estudo de caso, no qual se evidencia que o $85 \%$ da mostra acha-se em níveis de regular a deficiente em leto-escritura, classificando os estudantes no nível A1 de competência no domínio da sua língua materna de acordo com os standards do Marco Comum Europeu.

Palavras-chave: competências, leto-escritura, pós-graus, Educação Superior.

\section{Introducción}

El Proyecto Educativo Institucional (PEI) (2012) de la Escuela de Posgrados de la Fuerza Aérea Colombiana (EPFAC) afirma que el proceso de formación de los estudiantes ha de conducir al desarrollo de competencias profesionales para el desempeño confiable del alumno, entre ellas se encuentran las siguientes:

Cognitivas, Investigativas, Tecnológicas, Militares, Comunicativas y Valorativas (PEI-EPFAC; 2012). Teniendo en cuenta el anterior planteamiento, el presente proyecto de investigación se centrará en la competencia comunicativa, específicamente, en lo concerniente al proceso de lectoescritura; toda vez que la producción de conocimiento disciplinar se construye a través de la escritura y se divulga a través de la lectura de dichos conocimientos.

De igual manera el PEI de la institución establece que las competencias comunicativas, son el punto de partida del conocimiento, debido a que cumplen un propósito fundamental en la escuela, dado que se convierten en el medio más adecuado para la generación, difusión e interiorización de la cultura militar aeronáutica.
El mismo documento afirma que el alumno Fuerza Aérea debe estar en aprendizaje continuo, para un estructurado desarrollo profesional y ello obliga al diseño de propuestas educativas pertinentes e innovadoras, con énfasis en el fortalecimiento de las competencias profesionales, operativas, humanas, técnicas y administrativas para impulsar los altos niveles de empoderamiento que sus crecientes responsabilidades le implican (PEI-EPFAC; 2012).

No obstante y pese a los planteamientos del PEI, se presenta como dificultad para la graduación de los especialistas de Logística Aeronáutica en la EPFAC; el cumplimiento del requisito: trabajo de investigación; ya que este exige de los estudiantes, no solamente un buen nivel de desarrollo de las competencias en lectoescritura, sino del desarrollo de competencias investigativas y profundos conocimientos en el campo disciplinar, en este caso, la Logística Aeronáutica.

Se evidencia en la información consignada en la tabla 1, en la columna 5 de izquierda a derecha, titulada: Estudiantes pendientes por graduar, el número de estudiantes no graduados, por cohorte de la especialización de Logística Aeronáutica, cohorte I a la XIII: 


\section{PX GRADO}

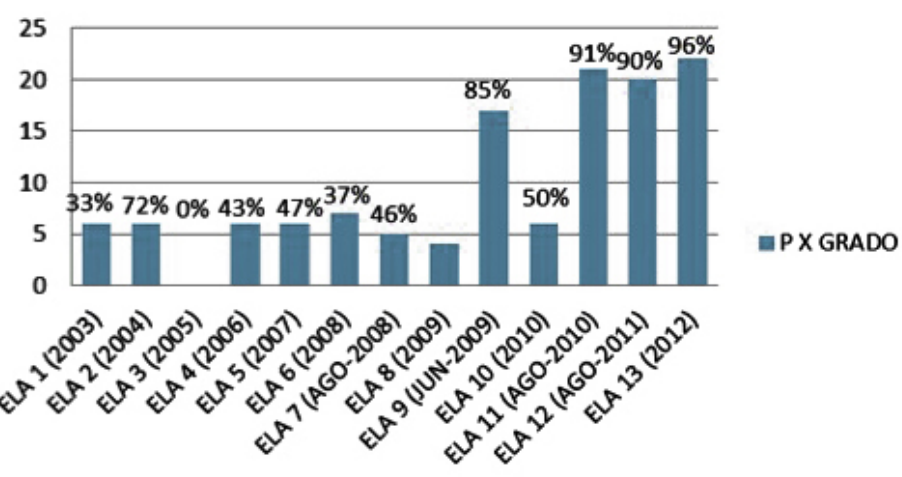

Figura 1. Estudiantes pendientes por grado ELA I a XIII. Fuente: la autora.

Desde el inicio de la primera cohorte de ELA, en el año de 2.003, a 2013, cohorte XIII, el porcentaje de graduados equivale al 35\%, ya que de 264 estudiantes que han cursado la especialización, se han graduado, 114 estudiantes; esto indica que, de 246 estudiantes que han cursado la especialización, 150, no se han graduado; presentando un índice de no graduados, equivalente al $65 \%$; evidenciándose como problemáticas para cumplir con los requisitos de graduación: el aplazamiento tanto de materias, como de ciclos y la no realización del trabajo de investigación.

Con base en lo anterior se construyen tanto el objetivo general: realizar el diagnóstico de las competencias lectoescritas de estudiantes de posgrado; como la hipótesis del presente trabajo de investigación, la cual se plasma en el siguiente interrogante: ¿es probable que el alto índice de no graduados en la especialización de Logística Aeronáutica, de la EPFAC, por falta de la realización del trabajo de investigación, se deba al bajo nivel del desarrollo de las competencias en lectoescritura de los estudiantes?

\section{Fundamentación teórica}

Resulta importante anotar en este punto, que se trabaja al interior de la investigación, el término competencia, como lo define la Organización para la Cooperación y el Desarrollo Económico (OCDE):
Una competencia es más que conocimientos y destrezas. Involucra la habilidad de enfrentar demandas complejas, apoyándose en y movilizando recursos psicosociales (incluyendo destrezas y actitudes) en un contexto en particular. Por ejemplo, la habilidad de comunicarse efectivamente, es una competencia que se puede apoyar en el conocimiento del lenguaje de un individuo, destrezas prácticas en tecnología e información y actitudes con las personas que se comunica (Rychen, et al, 2006).

Para la OCDE, la competencia lectora es: la capacidad de comprender, utilizar, reflexionar e interesarse por los textos escritos para alcanzar los propios objetivos, desarrollar el conocimiento, potencial personal y participar en la sociedad (OCDE; 2010:14). Un profesional del siglo XXI debe poseer diversas competencias; pero una condición fundamental es la competencia en lectoescritura, ya que a través de ella es que se tiene acceso al conocimiento.

\section{Pedagogía, didáctica y lingüística aplicadas a la enseñanza de la lectoescritura}

Desde 1986 el área de didáctica de la lengua se cuenta entre las que componen, formalmente, el panorama de materias de la universidad, Algunas décadas antes, diversos investigadores trabajaban para hacer posible este hecho: Hymes (1971), fundaba el concepto de competencia comunicativa; Hallyday (1979) aportaba estudios fundamentales para constituir científicamente la didáctica de la lengua.

Mendoza (1998) afirma en la introducción de Conceptos clave en Didáctica de la Lengua: "El marco de ésta área se ha ido definiendo con aportaciones múltiples, basadas y seleccionadas según criterios de transposición didáctica que confirieran coherencia y funcionalidad a la actividad base de la didáctica de la lengua a partir del marco de referencia delimitado por supuestos lingüístico-comunicativos, estético-receptivos, cognitivoconstructivistas y pragmáticos; la didáctica de la lengua ha comenzado a hacer notar sus aportaciones en el sistema educativo y en la actividad escolar, orientadas a la doble perspectiva de la enseñanza y el aprendizaje”. 
La práctica de la didáctica de la lectoescritura en el aula, requiere de una base teórica sólida mediante la cual se pueda aplicar la enseñanza, para su aprendizaje por parte de los estudiantes, esa teoría, se compone de unas disciplinas implicadas en ello: la lingüística, la pedagogía y psicología del aprendizaje.

La lingüística proporciona el campo de estudio, así como los contenidos y ejercicios de manipulación de la lengua, indispensables para que el aprendizaje siga su adecuada progresión, la pedagogía y la didáctica, le brindan al docente las acciones y herramientas a través de las cuales planifica, evalúa y ejecuta su enseñanza; por último, es la psicología del aprendizaje la disciplina que proporciona los procesos psíquicos o mentales que ocurren en el cerebro a la hora de aprender la lengua, por tales razones, la didáctica de la lectoescritura, mantiene una estrechez relacional con los saberes lingüísticos, tales como la lingüística general, lingüística aplicada, lingüística estructural, lingüística pragmática, lingüística cognitiva, lingüística textual, sociolingüística o psicolingüística, entre otras y con las disciplinas de las ciencias de la educación tales como la pedagogía, psicopedagogía, didáctica general y didácticas específicas

\section{Metodología}

\section{Tipo de investigación: Estudio de caso}

Teniendo en cuenta el planteamiento de Tamayo; (1997) frente a la conceptualización del estudio de caso: "este tipo de investigaciones tienen como características el estudio en profundidad de una unidad de observación, teniendo en cuenta características y procesos especificos o el comportamiento total de esa unidad en su ciclo de vida total o un segmento de ella". La presente investigación, se enmarca en el estudio de caso; pese a que plantea una problemática general de la educación, como la apropiación de las competencias en lectoescritura; pero estudiándola en profundidad, en el contexto militar, en el nivel de estudio de especialización y tomando una muestra específica, de la especialización de Logística Aeronáutica de la Escuela de Posgrados de la FAC.
Etapa 1: Enunciar el objetivo de la investigación. Realizar el diagnóstico del desarrollo de las competencias en lectoescritura, de los estudiantes de Posgrado de la EPFAC.

\section{Etapa 2: Indicar la unidad de estudio del caso, sus} características, relaciones y procesos observables. En esta etapa se presenta una unidad de estudio general y otra especifica; la primera unidad de estudio la componen 162 estudiantes no graduados de la ELA; desde 2003 a 2013, con quienes se determinó a través de encuesta; cuáles son los factores de mayor incidencia; en la no elaboración del trabajo de investigación, como requisito para el grado, los estudiantes son oficiales y civiles de la FAC y del ámbito aeronáutico, quienes cursan la especialización, la edad promedio oscila entre los 30 y 32 años, la muestra se compone de hombres y mujeres; a quienes se les aplicó una encuesta, la cual considera tres variables a saber: competencias en lectoescritura, competencias investigativas y el tiempo; a fin de determinar las posibles causas por las cuales no han realizado el trabajo de investigación.

La unidad de estudio específica es una cohorte de la especialización de Logística Aeronáutica; en la cual se mide el estado de las competencias lectoescritas; en cuanto a las características, procesos y relaciones a observar, estas se encuentran establecidas en el examen de español, propuesto por el marco común europeo, C2; en el cual se le evalúa al estudiante: el uso de la lengua, la compresión de lectura y la expresión escrita; por otro lado analizando las pruebas presentadas por los estudiantes, se puede establecer la relación entre el desarrollo de la comprensión de lectura, con el proceso de escritura.

Etapa 3: Técnicas de Recolección de Información. Las técnicas de recolección utilizadas fueron cuestionarios; éstos se enfocaron a acopiar dos tipos de información, por una parte; diagnosticar las causas, por las cuales el índice de no graduados de ELA es del 70\%. Presentando un mayor porcentaje de no graduados, debido al incumplimiento del requisito: trabajo de investigación. Para lo cual se diseñó como instrumento una encuesta, en la cual se presentan diversos factores que inciden en la no realización del 
trabajo de grado; dicha encuesta se aplicó a través de la plataforma Blackboard de la Escuela; por lo tanto, el estudiante la diligenció en línea; la plataforma realiza la tabulación de la misma. Con esta técnica se evidenció, cuál es el mayor factor de incidencia, que no les permite a los estudiantes cumplir con este requisito de graduación. La otra información recolectada, el nivel de desarrollo de las competencias en lectoescritura; que presentan los estudiantes de ELA; para ello se realizó la adaptación del examen C2 de español, estipulado por el Marco Común Europeo y se les aplicó a 21 estudiantes de posgrado de ELA; otorgándoles 120 minutos de tiempo para el desarrollo total de la prueba.La tabulación de este instrumento se realizó de manera manual, analizando los datos recogidos, los cuales se dividen en tres variables: uso de la lengua; comprensión lectora y expresión escrita.

\section{Instrumentos de recolección de información}

Encuesta: instrumento que consta de 20 preguntas, tendientes a indagar las posibles causas por las cuáles, el $70 \%$ de los estudiantes de ELA no se han graduado; en el cual se contemplan las variables: nivel de competencias investigativas, nivel de competencias en lectoescritura y tiempo. La encuesta se compone de 12 preguntas con graduación en dirección unipolar, 3 de respuesta múltiple, 2 de ordenamiento en rango, 2 dicotómicas y 1 pregunta, con única respuesta de acuerdo con la clasificación que establece Briones (1996), para el diseño de encuestas.

Prueba de lectoescritura: la prueba fue adaptada a partir del examen de español C2, establecido por el marco Común Europeo; esta prueba, consta de tres subpruebas: Subprueba 1: comprensión lectora. Ésta se compone de dos textos; el primer texto indaga sobre el nivel de lectura literal. El segundo más extenso que el anterior; de igual manera consta de 10 preguntas e indaga sobre el nivel de lectura inferencial; posee un lenguaje mucho más denso, a diferencia del primero.

Subprueba 2: expresión escrita. Ésta solicita al estudiante redactar un texto, presentándole tres opciones de tipos de texto diferente; utilizando de 15 a 20 líneas, con tono y el estilo adecuado: opción1: carta empresarial; cuyo objetivo es realizar una solicitud; opción 2: carta personal; de carácter argumentativo; opción 3: texto periodístico; de carácter diferenciador.

Subprueba 3: gramática y vocabulario. Ésta indaga el conocimiento formal de la lengua en cuanto a pronombres proposiciones adjetivos verbos y palabras con acento diacrítico.

Etapa 4: análisis de la información Los resultados se presentan de la siguiente manera: se consolidan estos resultados en cuadro general; para determinar de cuánto a cuánto oscilan los porcentajes por cada uno de los aspectos indagados; de las primeras 12 preguntas; seguidamente se presentan las gráficas con las preguntas 1 a 20, de las cuales se hace el análisis; posteriormente aparece la tabla de tabulación general de la prueba de lectoescritura; de la cual se realiza por cada columna la tabulación de cada subprueba y el análisis de las mismas.

\begin{tabular}{|c|c|c|c|c|c|c|c|c|c|c|c|c|}
\hline & $\begin{array}{l}5 \\
\frac{5}{2} \\
\frac{\pi}{5} \\
0 \\
0 \\
0 \\
0\end{array}$ & 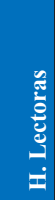 & $\begin{array}{l}\bar{\sigma} \\
\bar{\sigma} \\
\dot{5}\end{array}$ & 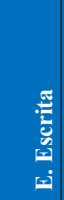 & 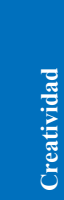 & 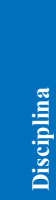 & 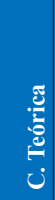 & 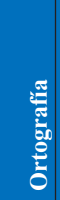 & 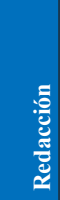 & 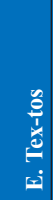 & 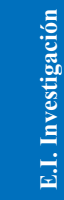 & 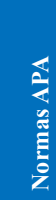 \\
\hline Muy bueno & 54,55 & 9,09 & 18,18 & 9,09 & 45,46 & 36,36 & 0 & 18,18 & 0 & 0 & 0 & 9,09 \\
\hline Bueno & 27,27 & 54,55 & 45,46 & 36,36 & 36,36 & 45,46 & 54,55 & 54,55 & 54,55 & 63,64 & 54,55 & 27,27 \\
\hline Regular & 0 & 27,27 & 18,18 & 36,36 & 9,09 & 0 & 18,18 & 9,09 & 36,36 & 18,18 & 36,36 & 18,18 \\
\hline Insuficiente & 0 & 0 & 0 & 0 & 0 & 0 & 0 & 0 & 0 & 0 & 0 & 9,09 \\
\hline Deficiente & 9,09 & 0 & 0 & 9,09 & 0 & 0 & 9,09 & 0 & 0 & 0 & 0 & 18,18 \\
\hline $\begin{array}{l}\text { Sin } \\
\text { contestar }\end{array}$ & 9,09 & 9,09 & 18,18 & 9,09 & 9,09 & 18,18 & 18,18 & 18,18 & 9,09 & 18,18 & 9,09 & 18,18 \\
\hline
\end{tabular}

Tabla1. Resultados encuesta en porcentajes

Fuente: la autora.

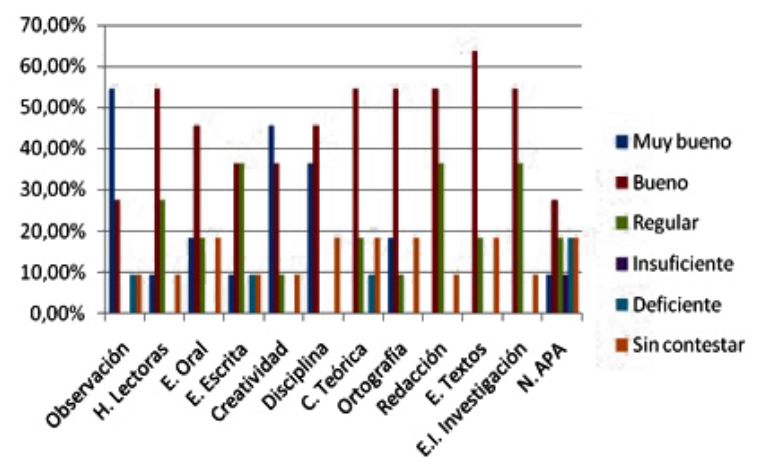

Figura 2. Resultados encuesta lectoescritura.

Fuente: la autora. 
Los resultados generales de la encuesta arrojan que entre el $9 \%$ y el $54 \%$ de los encuestados se consideran muy buenos en la mayoría de los 12 aspectos indagados; entre el 27 y el $63 \%$ se consideran buenos en todos los aspectos indagados; regulares entre el 9\% y el 36\%; insuficientes solo en el conocimiento de las normas APA el 9\%; deficientes entre el $9 \%$ y el $18 \%$ en cuatro aspectos y $\sin$ contestar entre el $9 \%$ y el $18 \%$ en todos los aspectos.

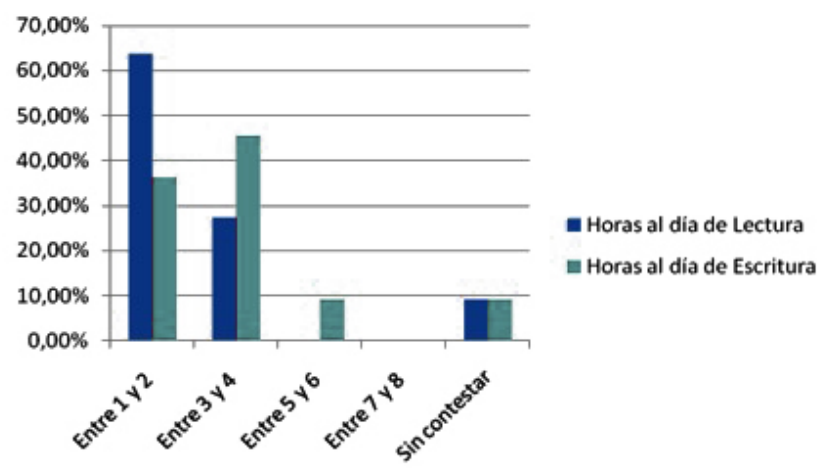

Figura 3. Horas dedicadas a la lectoescritura. Fuente: la autora.

Una de las variables sensibles del presente estudio es el tiempo dedicado a los procesos de lectoescritura, por parte de los estudiantes de ELA y es notorio que el $63 \%$ le dediquen a la lectura entre 1 y 2 horas diarias, sin especificar si es una lectura juiciosa, académica, con diccionario y técnicas de aprendizaje, como el subrayado y la nota al pie entre otras; o es una lectura informativa, de recreación o casual y sobre cuáles temas, si los propios de su trabajo de grado o de temas laborales, informativos o de recreación.

El 44\% afirma que dedica entre 3 y 4 horas diarias a la escritura; es decir que escribiendo 28 horas semanales, es probable terminar el trabajo de grado, en el tiempo estipulado por la EPFAC, no obstante esta encuesta se aplicó a estudiantes que justamente no lo han terminado, de lo que se infiere que hay una gran probabilidad de que esas horas dedicadas a la escritura, no todas sean académicas y específicas para el desarrollo del trabajo.

Esta gráfica muestra que aproximadamente el 45\% de la población medida, no trabaja sus procesos lectoescritos.

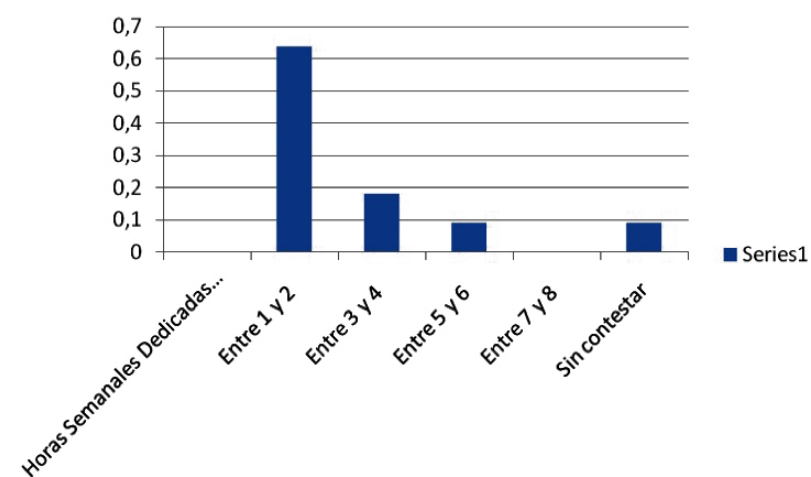

Figura 4. Horas semanales dedicadas a la investigación. Fuente: la autora.

Otra variable importante de tener en cuenta, es el tiempo con el cual cuentan los oficiales de posgrado de la FAC, para el desarrollo de sus investigaciones, de acuerdo con las respuestas dadas el $62 \%$ dedica entre 1 y 2 horas semanales a la investigación; lo anterior confirma que evidentemente lo respondido en el desarrollo de las habilidades de lectoescrtitura, no aplica para el desarrollo del trabajo de grado a presentar en la EPFAC.

Realmente es irrisorio dedicar entre 1 y 2 horas semanales a procesos de investigación, ya que el avance es muy lento y se amplía el porcentaje de probabilidad, de que al término de la investigación haya obsolescencia del conocimiento presentado; teniendo en cuenta que el área de conocimiento es la Logística Aeronáutica y ésta es muy dinámica.

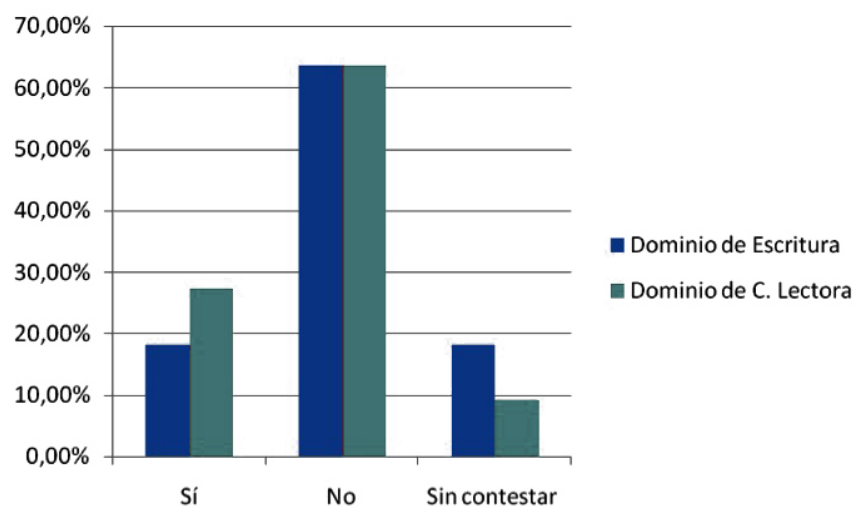

Figura 5. Dominio de procesos.

Fuente: la autora. 
Solamente un 9\% de los encuestados, dedica entre 5 y 6 horas semanales a la investigación y ese porcentaje, se corresponde aproximadamente, con el número de graduados de cada cohorte.

No obstante a lo respondido anteriormente por los estudiantes, el $63 \%$ manifiesta no poseer dominio sobre los procesos de lectoescritura, mientras el 18\% afirma poseer dominio sobre el proceso de escritura y el $28 \%$ sobre el proceso de comprensión lectora.

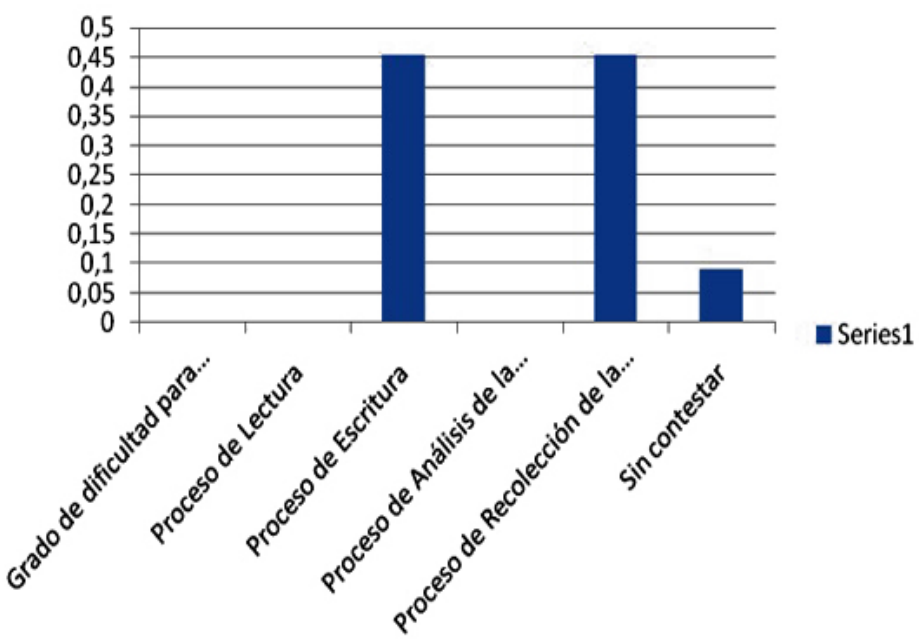

Figura 6. Grado de dificultad de los procesos.

Fuente: la autora.

Frente a la dificultad de los subprocesos que forman parte del proceso de investigación, el $45 \%$ de los estudiantes encuestados, manifiestan por unanimidad dificultad en el procesos de recolección de la información y el proceso de escritura; lo cual apunta como los resultados anteriores a evidenciar que hay falencias en el proceso lectoescrito de los estudiantes.

La tabla 2 muestra los resultados de la aplicación de la prueba de lectoescritura a 21 estudiantes de ELA; de izquierda a derecha, la primera columna muestra el número asignado a cada estudiante y el total de estudiantes; la columna dos presenta los resultados de la subprueba 1, la cual se compone de 10 puntos y fue evaluada en escala cuali-cuantitativa de 1 a 10 ; el nivel evaluado en esta prueba es el literal de lectura, donde el estudiante debe comprender explícitamente las ideas que expone la lectura.

\begin{tabular}{|c|c|c|c|c|c|c|c|c|c|c|}
\hline \multirow{4}{*}{$\begin{array}{l}\text { \# ESTUDI- } \\
\text { ANTES }\end{array}$} & \multicolumn{10}{|c|}{ TABULACIÓN PRUEBA DE LECTOESCRITURA } \\
\hline & \multirow{3}{*}{\begin{tabular}{|l|} 
SP1 \\
CALF \\
\end{tabular}} & \multirow{3}{*}{\begin{tabular}{|l|} 
SP2 \\
CALF \\
\end{tabular}} & \multirow{3}{*}{\begin{tabular}{|l|} 
SP3 \\
CALF \\
\end{tabular}} & \multirow{2}{*}{\multicolumn{7}{|c|}{\begin{tabular}{|l|} 
SUBPRUEBA N ${ }^{\circ} 4$ \\
EXPRESIÓN ESCRITA
\end{tabular}}} \\
\hline & & & & & & & & & & \\
\hline & & & & ESTRU & REDA & $\% \mathrm{E}$ & CAL & ORTO & $\% \mathrm{E}$ & CALF \\
\hline 1 & 8 & 4 & 6 & 5 & $0 / 18$ & $0 \%$ & 10 & $12 / 18$ & $67 \%$ & 2.3 \\
\hline 2 & 10 & 8 & 7 & 6 & $7 / 22$ & $32 \%$ & 6.8 & $12 / 22$ & $54 \%$ & 4.6 \\
\hline 3 & 6 & 6 & 8 & 4 & $1 / 8$ & $1 \%$ & 9 & $8 / 8$ & $100 \%$ & 0.0 \\
\hline 4 & 8 & 4 & 8 & 5 & $4 / 15$ & $27 \%$ & \begin{tabular}{|l|}
7.3 \\
\end{tabular} & $5 / 15$ & $33 \%$ & 6.7 \\
\hline 5 & 7 & 6 & 9 & 10 & $3 / 20$ & $15 \%$ & 8.5 & $7 / 20$ & $35 \%$ & 6.5 \\
\hline 6 & 7 & 4 & 9 & 5 & $6 / 16$ & $37.5 \%$ & \begin{tabular}{|l|}
6.2 \\
\end{tabular} & \begin{tabular}{|l|}
$14 / 16$ \\
\end{tabular} & $87.5 \%$ & 1.2 \\
\hline 7 & 6 & 5 & 8 & 5 & $8 / 20$ & $40 \%$ & \begin{tabular}{|l|}
6.0 \\
\end{tabular} & $14 / 20$ & $70 \%$ & 3.0 \\
\hline 8 & 5 & 3 & 8 & 5 & $2 / 11$ & $18 \%$ & 8.2 & $13 / 11$ & $100 \%$ & 0 \\
\hline 9 & 9 & 7 & 7 & 4 & $6 / 19$ & $32 \%$ & \begin{tabular}{|l|}
6.8 \\
\end{tabular} & $4 / 19$ & $21 \%$ & 7.9 \\
\hline 10 & 8 & 5 & 10 & 10 & $0 / 12$ & $0 \%$ & 10 & $0 / 12$ & $0 \%$ & 10 \\
\hline 11 & 7 & 5 & 9 & 4 & $4 / 18$ & $22 \%$ & 7.8 & $9 / 18$ & $50 \%$ & 5 \\
\hline 12 & 5 & 7 & 5 & 5 & $9 / 19$ & $47 \%$ & 5.3 & $9 / 19$ & $47 \%$ & 5.3 \\
\hline 13 & 8 & 4 & 10 & 5 & $2 / 9$ & $20 \%$ & 8 & $0 / 9$ & $0 \%$ & 10 \\
\hline 14 & 6 & 6 & 6 & 7 & $6 / 16$ & $38 \%$ & 6.2 & $21 / 16$ & $76 \%$ & 0.0 \\
\hline 15 & 5 & 6 & 7 & 7 & $1 / 18$ & $5.5 \%$ & 9 & $6 / 18$ & $33 \%$ & 7.7 \\
\hline 16 & 10 & 5 & 10 & 3 & $3 / 16$ & $19 \%$ & \begin{tabular}{|l|}
8.1 \\
\end{tabular} & $7 / 16$ & $44 \%$ & 6.6 \\
\hline 17 & 8 & 5 & 7 & 6 & $12 / 19$ & $63 \%$ & 2.7 & \begin{tabular}{|l|}
$14 / 19$ \\
\end{tabular} & $74 \%$ & 2.6 \\
\hline 18 & 8 & 4 & 7 & 3 & $8 / 14$ & $57 \%$ & 4.3 & $9 / 14$ & $64 \%$ & 3.6 \\
\hline 19 & 7 & 4 & 9 & 10 & $0 / 21$ & $0 \%$ & \begin{tabular}{|l|}
10 \\
\end{tabular} & $1 / 21$ & $99 \%$ & 9 \\
\hline 20 & 9 & 3 & 8 & 5 & $5 / 20$ & $25 \%$ & 7.5 & $10 / 20$ & $50 \%$ & 5 \\
\hline 21 & 7 & 4 & 8 & 6 & $5 / 17$ & $70 \%$ & 7 & $\begin{array}{l}11 / 17 \\
\end{array}$ & $25 \%$ & 2.5 \\
\hline $\begin{array}{l}\mathrm{N}^{\circ} \mathrm{DE} \\
\text { ESTUDIANTES }\end{array}$ & \begin{tabular}{|l}
$\frac{1}{L}$ \\
$\frac{d}{W}$ \\
崖 \\
\end{tabular} & 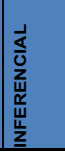 & 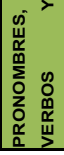 & 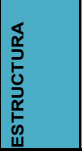 & 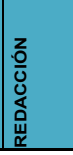 & 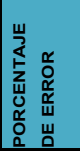 & 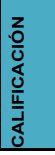 & \begin{tabular}{|l}
$\frac{5}{4}$ \\
$\frac{1}{5}$ \\
$\frac{d}{0}$ \\
0 \\
$\frac{0}{0}$ \\
0 \\
\end{tabular} & 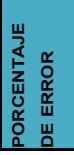 & 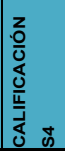 \\
\hline
\end{tabular}

Tabla 2. Tabulación prueba lectoescritura. Fuente: la autora.

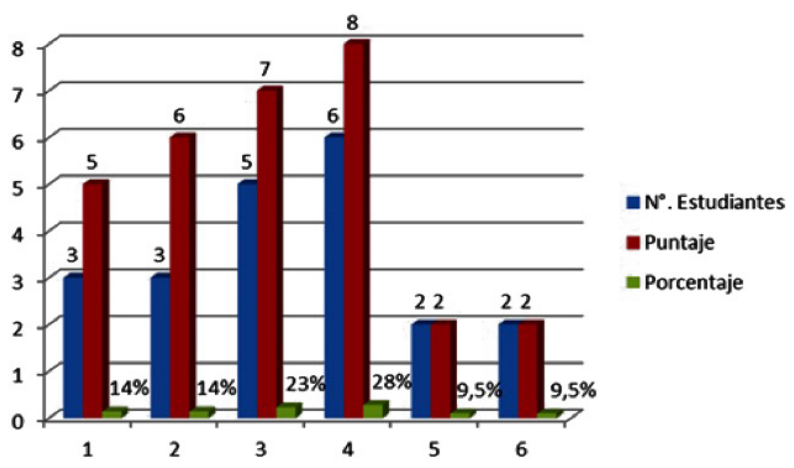

Figura 7. Puntaje subprueba 1.

Fuente: la autora.

El puntaje mínimo de aprobación es de 6/10; por lo tanto, en la subprueba 1, se determina que de los 21 estudiantes que la presentaron, aprobaron 18, es decir, el $85.7 \%$ aprobó; no obstante, aunque el porcentaje refleja un gran número de estudiantes aprobados, lo que podría hacer pensar que se encuentran en buen nivel; el análisis arroja lo contrario, pues el nivel de lectura literal, para posgrado debe tener un porcentaje del $100 \%$; esto en realidad determina que tan sólo dos estudiantes, de los 
21 evaluados, se encuentran en el nivel esperado y los 19 restantes se encuentran por debajo del nivel, en la siguientes proporciones:

Del total de los estudiantes, tres no la aprobaron, es decir el $14.3 \%$; podría pensarse que es un resultado favorable, pero el nivel literal de lectura es básico y por tanto estos estudiantes por ser de posgrado, deberían haber pasado la prueba todos y con un puntaje del $100 \%$.

El 14\% de los estudiantes reprobó con un puntaje de 5, el $14 \%$ aprobó con puntaje mínimo de 6 , el 24\% presentan un nivel regular, el $29 \%$ un buen nivel, el $9.5 \%$ muy bueno y el 9.5\% excelente. Es decir que de 21 estudiantes, diez se encuentran en los niveles de bueno a excelente; cuando todos los 21 como ya se dijo, por ser estudiantes de posgrado deberían estar en el nivel excelente.

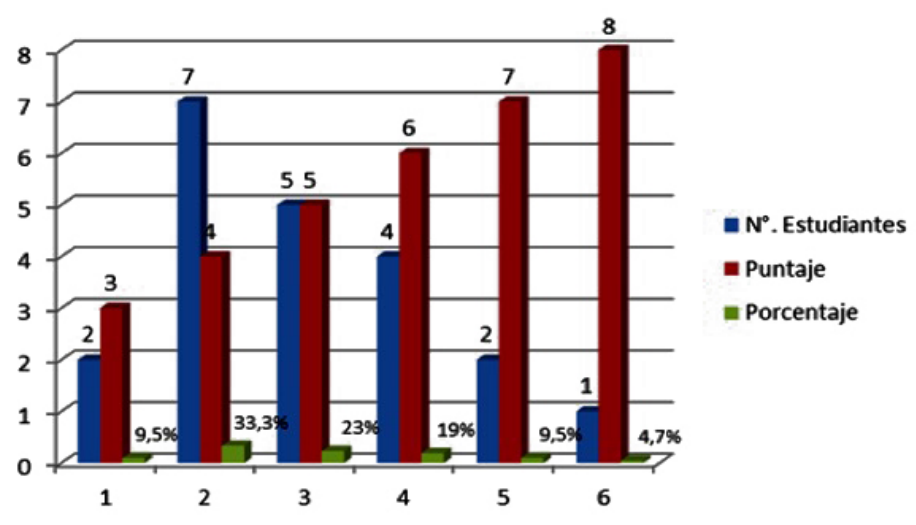

Figura 8. Puntaje subprueba 2 Fuente: la autora.

En la subprueba 2 se determina que de los 21 estudiantes que la presentaron, aprobaron 7, es decir el 33.3\%; aprobó y el $66.7 \%$ No aprobó es decir que el nivel de lectura inferencial de los estudiantes en esta prueba, no llega a la mitad, lo cual indica que se debe dar atención y refuerzo a este nivel de lectura, en los estudiantes de posgrado; ya que en este nivel de lectura, los estudiantes de posgrado debe tener un porcentaje del 100\% y tan solo 7 de los 21 evaluados está en el 33\%, nivel regular, es decir que el $66.7 \%$ está en un nivel insuficiente; sin alcanzar el nivel bueno o excelente.
Del total de los estudiantes, 14 no la aprobaron, es decir el 66.6\%; resultado desfavorable, pues el nivel inferencial de lectura para posgrado, debe encontrarse en el $100 \%$. El 9.5\% de los estudiantes reprobó con un puntaje de 3, el 33.3\% reprobó con puntaje de 4, el 23\% reprobó con puntaje de 5. Es decir que de 21 estudiantes, ninguno se encuentra en los niveles de bueno a excelente; cuando todos los 21 como ya se dijo, por ser estudiantes de posgrado deben estar en el nivel excelente.

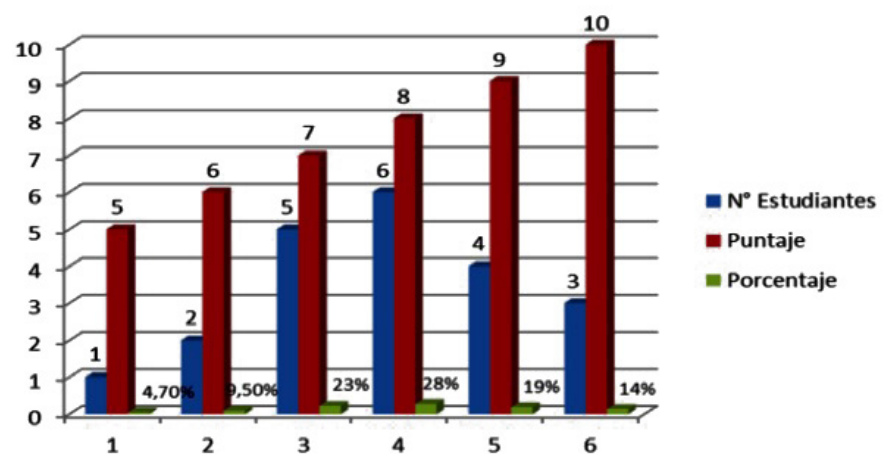

Figura 9. Resultados subprueba 3

Fuente: la autora.

En la subprueba 3 se determina que de los 21 estudiantes que la presentaron, aprobaron 20, es decir el 95\%; No aprobó el 5\% es decir que el nivel de escritura de los estudiantes en esta prueba, cumple con el nivel que se debe tener en posgrado; no obstante se debe tener en cuenta que estudiantes de posgrado debe tener un porcentaje y nivel del $100 \%$, en el desarrollo de la escritura; por lo tanto se analizan los puntajes obtenidos, 2 de los 21 evaluados obtuvieron puntaje mínimo o aceptable, es decir el 9.5\%, 5 obtuvieron puntaje 7 , es decir regular, equivalente al $23 \%, 6$ estudiantes obtuvieron puntaje 8 , es decir bueno, equivalente al 28\%; 4 estudiantes obtuvieron puntaje 9 , es decir muy bueno equivalente al 19\% y solo 3 alcanzaron nivel excelente es decir el 14\%; de lo cual se puede afirmar que el $67 \%$ no se encuentra en el nivel esperado, he aquí, otro punto importante para fortalecer.

Para evaluar la prueba escrita se tuvieron en cuenta 3 factores importantes en el momento de la escritura: estructura del escrito, redacción y ortografía, por lo cual se presentaran cada uno de estos aspectos evaluados de manera individual. 
Diagnóstico de las competencias lectoescritas en estudiantes de posgrado

\begin{tabular}{|c|c|c|}
\hline $\begin{array}{c}\text { Número } \\
\text { de } \\
\text { Estudiantes }\end{array}$ & Puntaje & Porcentaje \\
\hline 2 & 3 & $9.5 \%$ \\
\hline 3 & 4 & $14 \%$ \\
\hline 8 & 5 & $38 \%$ \\
\hline 3 & 6 & $14 \%$ \\
\hline 2 & 7 & $9.5 \%$ \\
\hline 3 & 10 & $14 \%$ \\
\hline
\end{tabular}

Tabla 3. Estructura prueba escrita.

Fuente: la autora.

Cuando se evalúa la estructura de un escrito, se hace referencia de acuerdo con su género y su tipo, narrativo expositivo, explicativo, descriptivo; si la escritura se corresponde con el mismo; con base en lo anterior, los estudiantes crearon en su prueba escrita, tres tipos de texto: carta formal, carta comercial y artículo; cada uno de estos textos posee su estructura y características particulares, las cuales fueron evaluadas en la estructura, de la siguiente manera: de los 21 estudiantes que presentaron la prueba, 8 la aprobaron, es decir el $37.5 \%$ y 13 no la aprobaron es decir el $62.5 \%$.

No obstante de ese porcentaje aprobado, el 14\% se encuentra en un nivel aceptable, el $9.5 \%$ en regular y el $14 \%$ en excelente; es decir que se hace necesario, crear propuestas, mecanismos, estrategias, para fortalecer los procesos de escritura de los estudiantes de posgrado y del país en general, para lograr el desarrollo requerido en este nivel de estudios.

La prueba de redacción se evaluó de acuerdo con la producción escrita de cada estudiante; en la prueba se indicaba crear un texto de 15 a 20 renglones, ya fuese una carta comercial, personal o un artículo; no obstante la producción osciló entre 8 a 22 renglones, de los diferentes tipos de textos mencionados, 4 de los 21 estudiantes evaluados realizaron producción escrita de acuerdo con lo solicitado, es decir el 19\%, mientras que el 81\% realizó una producción menor a la solicitada.

\begin{tabular}{|c|c|c|}
$\begin{array}{c}\text { Renglones } \\
\text { Escritos }\end{array}$ & $\begin{array}{c}\text { Errores } \\
\text { encontrados }\end{array}$ & $\begin{array}{c}\text { Porcentaje } \\
\text { de Error }\end{array}$ \\
\hline 12 & 0 & $0 \%$ \\
\hline 18 & 0 & $0 \%$ \\
\hline 21 & 0 & $0 \%$ \\
\hline 8 & 1 & $1 \%$ \\
\hline 18 & 2 & $5.5 \%$ \\
\hline 11 & 2 & $18 \%$ \\
\hline 9 & 3 & $20 \%$ \\
\hline 16 & 3 & $19 \%$ \\
\hline 20 & 4 & $15 \%$ \\
\hline 18 & 4 & $22 \%$ \\
\hline 15 & 5 & $27 \%$ \\
\hline 20 & 5 & $25 \%$ \\
\hline 17 & 6 & $70 \%$ \\
\hline 16 & 6 & $37.5 \%$ \\
\hline 16 & 6 & $38 \%$ \\
\hline 19 & 7 & $32 \%$ \\
\hline 22 & 8 & $32 \%$ \\
\hline 14 & 8 & $57 \%$ \\
\hline 20 & 9 & $40 \%$ \\
\hline 19 & 12 & $47 \%$ \\
\hline 19 & & $63 \%$ \\
\hline
\end{tabular}

Tabla 4. Resultados prueba de redacción.

Fuente: la autora.

De esa producción, tres estudiantes obtuvieron $0 \%$ de error en redacción, es decir el 14\% de los estudiantes; 2 estudiantes obtuvieron 1, 2, 3, 4 y 5 error es decir el 9.5\%; tres estudiantes obtuvieron 6 errores, es decir el 14\% de la población, 1 estudiante obtuvo 7 errores es decir el 4.7\%, 2 estudiantes 8 errores, equivalentes al 9.5\%, un estudiante 9 errores, equivalente al $4.7 \%$ y un estudiante 12 errores, equivalente al 4.7\%. De acuerdo con lo presentado de la población; el $41 \%$ de los estudiantes evaluados, tiene un buen nivel de redacción y el $49 \%$ restante debe mejorarlo.

La evaluación del aspecto ortográfico también se realizó de acuerdo con la producción escrita de cada estudiante, es decir de acuerdo con el número de renglones escritos y los errores obtenidos en la totalidad del texto, para establecer el porcentaje de error, en su producción 


\begin{tabular}{|c|c|c|}
\hline $\begin{array}{c}\text { Renglones } \\
\text { Escritos }\end{array}$ & $\begin{array}{c}\text { Errores } \\
\text { encontrados }\end{array}$ & $\begin{array}{c}\text { Porcentaje } \\
\text { de Error }\end{array}$ \\
\hline 9 & 0 & $0 \%$ \\
\hline 12 & 0 & $0 \%$ \\
\hline 21 & 1 & $4.7 \%$ \\
\hline 19 & 4 & $21 \%$ \\
\hline 15 & 5 & $33 \%$ \\
\hline 18 & 6 & $33 \%$ \\
\hline 20 & 7 & $35 \%$ \\
\hline 16 & 7 & $44 \%$ \\
\hline 8 & 8 & $100 \%$ \\
\hline 18 & 9 & $50 \%$ \\
\hline 19 & 9 & $47 \%$ \\
\hline 14 & 9 & $64 \%$ \\
\hline 20 & 10 & $50 \%$ \\
\hline 17 & 11 & $25 \%$ \\
\hline 18 & 12 & $67 \%$ \\
\hline 22 & 12 & $54 \%$ \\
\hline 11 & 13 & $100 \%$ \\
\hline 16 & 14 & $87.5 \%$ \\
\hline 20 & 14 & $70 \%$ \\
\hline 19 & 14 & $74 \%$ \\
\hline 16 & 21 & $76 \%$ \\
\hline
\end{tabular}

Tabla 5. Resultados pruebas de ortografía. Fuente: la autora.

escrita, 2 estudiantes obtuvieron $0 \%$ de error, ello equivale al $9.5 \%$, no obstante se debe tener en cuenta que la producción escrita de estos estudiantes fue inferior a la solicitada. 1 estudiante presentó 1 error, cumpliendo con la producción estipulada, equivale al porcentaje del 4.7\%. Entre 4, 5, 6, 8, 10, 11, 13 a 21, errores, fueron presentados también por 1 estudiante, equivaliendo cada uno de ellos a un $4.7 \%$ de la población total.

Tres estudiantes presentaron 9 y 14 errores, lo que equivale al $14 \%$ de la población, cada uno y 2 estudiantes presentaron 12 errores equivalentes al 9.5\% de la población, lo anterior evidencia, que el $28 \%$ de la población posee una producción escrita que oscila entre aceptable a bueno, sin alcanzar el nivel de excelencia, requerido, para posgrados; es decir que hay un $72 \%$ de la población, que necesita fortalecer el aspecto ortográfico.

\section{Etapa 6: Discusión de resultados en función de los objetivos propuestos al iniciar el estudio.}

En esta etapa de la investigación se realiza la presentación de los resultados generales del proceso de investigación y se someten a discusión, a fin de posteriormente poder realizar la propuesta que dé solución a la problemática, presentar conclusiones y recomendaciones; se inicia presentando las conclusiones generales de la encuesta y luego de la prueba realizada a los estudiantes, a fin de realizar la discusión.

\begin{tabular}{|c|c|c|c|c|}
\hline Ítemes & $\begin{array}{c}\text { Percepción } \\
\text { Estudiantes } \\
\text { Encuesta }\end{array}$ & $\begin{array}{l}\text { Nivele Cuali- } \\
\text { cuantitativos }\end{array}$ & $\begin{array}{c}\text { Resultados } \\
\text { Prueba }\end{array}$ & $\begin{array}{l}\text { Niveles Cuali- } \\
\text { cuantitativos }\end{array}$ \\
\hline $\begin{array}{l}\text { Proceso } \\
\text { general de } \\
\text { lectoescri- } \\
\text { tura }\end{array}$ & $50-60 \%$ & $\begin{array}{l}\text { Bueno } \\
80 \%\end{array}$ & $\begin{array}{l}4.7 \% \\
4.7 \% \\
4.7 \%\end{array}$ & $\begin{array}{l}\text { Excelente } \\
100 \% \\
\text { Muy Bueno } \\
90 \% \\
\text { Bueno } 80 \%\end{array}$ \\
\hline $\begin{array}{c}\text { Expresión } \\
\text { Escrita }\end{array}$ & $35 \%$ & $\begin{array}{l}\text { Bueno } \\
80 \%\end{array}$ & $4.7 \%$ & Bueno $80 \%$ \\
\hline Ortografía & $55 \%$ & $\begin{array}{l}\text { Bueno } \\
80 \%\end{array}$ & $0 \%$ & Bueno $80 \%$ \\
\hline Redacción & $55 \%$ & $\begin{array}{l}\text { Bueno } \\
80 \%\end{array}$ & $\begin{array}{l}4.7 \% \\
4.7 \% \\
14 \%\end{array}$ & $\begin{array}{l}\text { Excelente } \\
100 \% \\
\text { Muy Bueno } \\
90 \% \\
\text { Bueno } 80 \%\end{array}$ \\
\hline $\begin{array}{l}\text { Estructura } \\
\text { de textos }\end{array}$ & $65 \%$ & $\begin{array}{l}\text { Bueno } \\
80 \%\end{array}$ & $14 \%$ & $\begin{array}{l}\text { Excelente } \\
100 \%\end{array}$ \\
\hline
\end{tabular}

Tabla 6. Comparativo encuesta-prueba.

Fuente: la autora.

De acuerdo con los resultados arrojados en el proceso de investigación, se evidencia que una de las principales causas, para que no se realice el trabajo de investigación, de acuerdo con las perspectivas de los estudiantes son: la expresión escrita, el conocimiento teórico, las normas 
APA, proceso de lectoescritura, el tiempo y la recolección de la información. No obstante con el desarrollo de las prueba lectoescrita, se confirma que se debe mejorar ostensiblemente el desarrollo de las competencias lectoescritas de los estudiantes, no solamente a fin de fortalecer el desarrollo del trabajo de investigación, sino también el desempeño académico general, el desempeño en cada uno de sus cargos y en todos los aspectos de la vida, que se encuentran transversalizados por la lectoescritura.

El desarrollo de las competencias en lectoescritura es objeto de atención; toda vez que es mediante el desarrollo de las mismas que se tiene acceso al conocimiento; de acuerdo con la prueba presentada por los estudiantes de ELA, entre el 95\% y el $85 \%$ se encuentran en niveles de regular a insuficiente; por lo cual se hace necesario realizar: investigaciones, sistemas, planes, propuestas a nivel nacional, que permitan el desarrollo de los procesos lectoescritos desde el inicio de la escolarización hasta el postdoctorado; ya que de acuerdo con la globalización y las políticas internacionales que rigen, las competencias básicas, las comunicativas, las laborales, las académicas y las específicas de cada saber son los aspectos a desarrollar en los individuos, a fin de que se desempeñen en la sociedad.

Existen parámetros internacionales desde la OCDE y otros organismos, que propenden por el desarrollo de las competencias; a nivel nacional hay organismos como REDLEES, el Ministerio de Educación, la Universidad del Valle y las diferentes instituciones de educación de todos los niveles; cada una con planes propios y no cohesionados; que al igual que las instituciones internacionales, propenden por el desarrollo de la lectoescritura; en el ámbito militar, las fuerzas tienen el SEFA (2010) sistema Educativo de las Fuerzas Armadas, el PEFA (2008) proyecto educativo de las Fuerzas Armadas y específicamente la FAC, cuenta con el PEISEFAC (2013), documentos directrices del desarrollo educativo en las fuerzas; los cuales plantean el fortalecimiento del sistema educativo militar a través del desarrollo de las competencias del personal de las diferentes instituciones.

\section{Conclusiones}

Las investigaciones realizadas a nivel de educación superior con énfasis en la lectoescritura, en Colombia llegan a nivel de pregrado, de acuerdo con lo indagado en las bases de datos de las universidades nacionales, REDLEES y el PNL.

La percepción de la muestra de estudiantes de posgrado de ELA, consultados frente al desarrollo de sus competencias lectoescritas, es que entre el $50 \%$ y el $60 \%$ de la población considera que sus competencias se encuentran en $80 \%$ de desarrollo, lo cual equivaldría a un nivel $\mathrm{C} 1$ de competencia en lengua materna; no obstante la prueba de lectoescritura arrojó nivel A1.

Las causas por las cuáles, el $60 \%$ de los estudiantes de la Especialización de Logística aeronáutica, no han presentado su trabajo de grado, obedecen a que entre el 9 y el $36 \%$ de los estudiantes encuestados manifiestan dominar de manera aceptable los procesos de escritura, redacción y trabajo de escritorio en el desarrollo de investigación; adicional a ello hay una variable sensible y es que solamente un $9 \%$ de los encuestados, dedica entre 5 y 6 horas semanales a la investigación.

El estado de las competencias lectoescritas de los estudiantes de la especialización de logística aeronáutica es que el $15 \%$ de la muestra se encuentra en niveles de desempeño de la competencia lectoescrita entre excelente a muy bueno, es decir entre 100 y $80 \%$. Mientras el $85 \%$ de la muestra se encuentra en niveles de regular a deficiente, es decir en niveles de 60 a $10 \%$.

De acuerdo con la investigación realizada, los resultados arrojados y las conclusiones anteriores; se puede afirmar que se comprueba la hipótesis planteada, ya que justamente el alto índice de no graduados en la especialización de Logística Aeronáutica, de la EPFAC, plantean dificultad sobre todo con las competencias de escritura para la realización del trabajo de investigación. 
Los estudiantes de posgrados deben encontrarse en el nivel de dominio $\mathrm{C} 2$, de su primera lengua, de acuerdo con la clasificación planteada por el Marco Común Europeo, No obstante lo encontrado en la muestra permite clasificar a los estudiantes en un nivel A1.

En la academia a nivel de posgrado falta darle la importancia suficiente al desarrollo de las competencias en lectoescritura, ya que se asume que el estudiante de este nivel de estudios ya alcanzó el máximo nivel de las mismas; no obstante en este estudio se determina que no es así y que deben realizar acciones, para que el estudiante alcance o incremente el nivel de sus competencias en Lectoescritura.

Se cree que la enseñanza de la lectoescritura solo le compete al área de lenguaje; desconociendo que todas las áreas del conocimiento son permeadas por la lectoescritura. Las competencias lectoescritas, por formar parte del proceso de comunicación de los seres humanos; tienen gradaciones, las cuales se deben profundizar en los distintos niveles de educación, desde el preescolar hasta el postdoctorado.Se deben aunar esfuerzos para crear un sistema nacional de lectura y escritura, que permee todos los niveles educativos, a fin de que los esfuerzos de cada institución converjan en dicho plan y halla una articulación del desarrollo de las competencias en lectoescritura a nivel nacional, que a su vez responda a las expectativas de los estándares internacionales.

\section{Referencias}

Cassany, D. (1995). La cocina de la escritura. Ed 11ª Barcelona: Anagrama.

Fernández, et al. (2006). La tecnología de identificación por radiofrecuencia. http://dialnet.unirioja.es/servlet/ articulo? $\operatorname{codigo}=1448367$

Fielding, G (1952). The Frog Prince and Other Poems. http://gabrielfielding.com/id2.html

Halliday, M.A.K. (1979). El lenguaje como semiótica social. México: Fondo de Cultura Económica.

Hernández, Fernández y Baptista. (2006). Metodología de la investigación. México: McGraw-Hill.
Hymes, D. 1971. "Competence and performance in linguistic theory". En: Acquisition of languages: Models and methods. Ed. Huxley and E. Ingram. New York: Academic Press. 3-23.

Marco Común Europeo (2013). Referencia para las lenguas. http://www.europarl.europa.eu/ RegData/etudes/etudes//2013/495871/IPOL-CULT ET(2013)495871(SUM01)_ES.pdf

Mendoza Fillola, A. (coord.) (1998). Conceptos clave en Didáctica de la Lengua y la Literatura. Barcelona: SEDLL/ICE/Horsori.

OCDE (2006). Panorama de la Educación: Indicadores de la OCDE. http://www.oecd.org/edu/skills-beyondschool/37393626.pdf

OCDE (2010) Working Paper 21st Century Skills and Competences for New Millennium Learners in OECD Countries (EDU Working paper no. 41) (2010) http:// recursostic.educacion.es/blogs/europa/media/blogs/ europa/informes/Habilidades_y_competencias_siglo21_ OCDE.pdf.

Instituto Militar Aeronáutico (2012). PEI. Fuerza Aérea Colombiana. Bogotá: Colombia.

PEFA (2008). Proyecto Educativo de las Fuerzas Armadas. Colombia. Bogotá: Rasgo y Color.

Plan Estratégico del Sistema Educativo de las Fuerzas Armadas PESE (2007-2019). Bogotá: Rasgo y Color.

Proyecto Educativo Institucional del Sistema Educativo de la Fuerza Aérea Colombiana PEISEFAC (2013). Bogotá. Colombia.

Rychen y Salganik (2006). Las competencias clave para el bienestar personal, económico y social. Primera Ed. en español. En: http://www.deseco.admin.ch/bfs/ deseco/en/index/03/02.parsys.78532.downloadList.94248. DownloadFile.tmp/2005.dscexecutivesummary.sp.pdf. Consultado 15 de noviembre de 2011.

Sistema Educativo de las Fuerzas Armadas SEFA (2010). Bogotá: Rasgo y Color.

Triana, L. y Ulloa, K. (2009). Comunicación en Diferentes Escenarios. Bogotá: Ed. Espacio, Universidad Minuto de Dios. 